\title{
Une cité face aux crises
}

Les remparts de la fidélité, de Louis d'Orléans à Charles VII, d'après les comptes de forteresse de la ville d'Orléans (1391-1427)

\section{Françoise Michaud-Fréjaville}

\section{(2) OpenEdition}

12 Journals

Édition électronique

URL : https://journals.openedition.org/crm/724

DOI : $10.4000 / \mathrm{crm} .724$

ISSN : 1955-2424

Éditeur

Honoré Champion

Édition imprimée

Date de publication : 1 juin 2005

Pagination : 85-100

ISSN : 1272-9752

\section{Référence électronique}

Françoise Michaud-Fréjaville, "Une cité face aux crises », Cahiers de recherches médiévales [En ligne],

12 spécial | 2005, mis en ligne le 28 juin 2008, consulté le 15 décembre 2022. URL : http:// journals.openedition.org/crm/724; DOI : https://doi.org/10.4000/crm.724 


\section{间M}

\section{$-8-$ \\ Une cité face aux crises : \\ Les remparts de la fidélité, de Louis d'Orléans à Charles VII, d'après les comptes de forteresse de la ville d'Orléans (1391-1427)}

En l'année 1429, il y eut deux héroïnes vers lesquelles se tournèrent les regards et les espoirs de l'un et l'autre camp qui se partageaient alors le royaume de France : une femme et une ville. Ce fut en effet quand la nouvelle du siège d'Orléans parvint jusqu'à la châtellenie de Vaucouleurs que Jeanne d'Arc se sentit tous les courages pour accomplir sa mission, en allant dire qu'elle délivrerait la ville et que le Dauphin serait sacré.

$\mathrm{La}$ « grande et nottable ville et cite assise en passaige et frontiere sur la rivière de Loyre ${ }^{1}$ ", son univers urbain - portes, murs, barrières, faubourgs, fossés - et son univers mental - craintes, espoirs, petitesses, travail acharné, flatteries, labeur quotidien - nous sont en partie dévoilés grâce à une très belle série de comptes épargnée par les désastres qui ont, au cours des siècles, frappé la ville dans ses bâtiments, son sous-sol et ses archives.

Pour les quarante années précédant la dure épreuve qui vit la capitale du duc Charles «moult oppressée et travaillée [...], et en péril d'être mis[e] en la servitude et obéissance $^{2} »$ par les troupes du comte de Salisbury, sont conservés 24 registres, couvrant chacun deux ans dont 14 pour la seule "forteresse $»^{3}$. Depuis 1384 , les

${ }^{1}$ Arch. dép. Loiret, CC 546, fol. 1v, lettre du roi Charles VI (Paris, 21 nov. 1416) sur «l'appetissement» de la pinte.

Les références, sauf indications expresses, sont celles des Archives communales d'Orléans antérieures à 1790, série CC, finances, impôts et comptabilité, conservées aux Archives départementales du Loiret.

Je dois remercier le Centre Jeanne d'Arc qui m'a prêté les microfiches des comptes, et la photocopie de ceux qui ont été édités. Ma gratitude va aussi aux conservateurs des Archives départementales qui m'ont permis la consultation des originaux.

Les registres n'ont été paginés que tardivement ; à l'origine, seules les dépenses étaient numérotées, en chiffres romains, mandement par mandement.

${ }^{2}$ Enguerrand de Monstrelet, Chroniques, éd. L. Douët D'Arcq, Paris, 1860, (Société de l'Histoire de France), t. VI, II, chap. 59, p. 321.

\begin{tabular}{|l|l|l|l|}
\hline Années & Forteresse & Commune & Receveur \\
\hline $1391-1393$ & CC 537 & id. & P. de Saint-Mesmin \\
\hline $1400-1401$ & CC 538 & manque & Jean le Breton \\
\hline $1401-1403$ & CC 539 & CC 644 & Girard Groussard \\
\hline $1403-1405$ & CC 540 & manque & Jean Asset \\
\hline $1405-1407$ & CC 541 & CC 645 & Pierre de Soye \\
\hline $1407-1409$ & manque & CC 646 & Jean Boilève \\
\hline $1409-1411$ & CC 542 & CC 647 & Pierre de Mareau \\
\hline $1411-1413$ & CC 543 & manque & Berthaud Mignon \\
\hline
\end{tabular}

Cahiers de Recherches Médiévales (XII -XV s.), 12spé, 2005 
bourgeois d'Orléans peuvent se réunir, élire des procureurs, avoir un receveur, décider d'une imposition ${ }^{4}$ pesant non seulement sur la ville et ses faubourgs, mais aussi sur des paroisses qui sont de ses «prises et mises», comme Olivet ou Gémignys. L'emploi de ces revenus était soumis à une reddition de comptes devant un représentant de l'autorité, royale à l'origine puis ducale. Nous ne possédons que la comptabilité qui a suivi l'échange fait en juin 1392 par Louis, de son apanage de Touraine contre le duché d'Orléans, mais nous savons que pendant les années qui précédèrent, la ville leva des tailles affectées à une dépense précise ${ }^{6}$. Dès sa prise de possession, le nouveau duc envisagea la possibilité d'obtenir du roi la levée d'une aide pour la remise en état de la forteresse ${ }^{7}$. Il était bien évident pour le prince que l'accroissement de puissance ainsi acquis ne devait en rien obérer ses propres revenus; les Orléanais semblent avoir été moins convaincus. À partir de $1400^{8}$, et en raison des menaces liées à l'avènement des Lancastre", on finit par convenir de

\begin{tabular}{|l|l|l|l|}
\hline $1413-1415$ & CC 544 & CC 648 & Jean Chiefdail \\
\hline $1415-1417$ & CC 545 & CC 649 & Jean Martin \\
\hline $1417-1419$ & CC 546 & CC 650 & Gilet Baudry \\
\hline $1419-1421$ & CC 547 & manque & Jacquet Deloynes \\
\hline $1421-1423$ & CC 548 & CC 651 & Jean Martin \\
\hline $1423-1425$ & manque & manque & Jacquet Cormereau (a) \\
\hline $1425-1427$ & CC 549 & CC 652 & Jacquet Deloynes \\
\hline $1427-1429$ & manque & CC 653 & Etienne de Bourges \\
\hline $1429-1431$ & CC 550 & manque & Jean Hilaire \\
\hline
\end{tabular}

(a) $\mathrm{CC} 549$, fol. $6 \mathrm{v}^{\circ}:$ « Dudit Jacquet Cormereau, précédent receveur de ladicte ville, la somme de C XIX 1. X s. VII d.p.»

Cf., R. Boucher de Molandon, «Les comptes de ville d'Orléans des XIV et $\mathrm{XV}^{\mathrm{e}}$ siècles », Mémoires de la Société Archéologique et Historique de l'Orléanais, t. XVIII (1884), p. 15-46. ${ }^{4} \mathrm{BnF}$, ms. fr. 11988, Cartulaire de la ville d'Orléans, fol. $20 \mathrm{sq}$

${ }^{5}$ Saint-Marceau, Saint-Jean-le-Blanc, Saint-Martin-sur-Leret, Saint-Hilaire-Saint-Mesmin, Saint-Pryvé, Saint-Loup-Saint-Marc, Saint-Jean-de-la-Ruelle, Champs et Gémigny (CC 538, fol. 3). Les paroisses de Saint-Hilaire-Saint-Mesmin et de Saint-Martin-sur-Leret (Olivet) intentèrent et perdirent un procès contre Orléans. Elles demandaient à payer moins d'impôts parce qu'en dépit de l'aspect riche des campagnes, les habitants étaient très pauvres, les terres étant possédées par des habitants de la ville (BnF, ms. fr. 11988, fol. 84, 1380).

${ }^{6} \mathrm{CC}$ 537, fol. 4 : taille de 1389 pour traiter le différend entre la ville et l'Université ; ibid., fol. 6 : taille de 1390 pour le capitaine; ibid., fol. 2 : taille de 1391 pour la venue du roi et du duc ; ibid., fol. 3 : taille de 1392 pour la forteresse et la venue du duc.

${ }^{7}$ CC 537, XXXIV : (nov. 1392) «à Jehan Asset et Jehan le Texier, procureurs de la ville [..,] pour un voyage par eulx faict à Paris, ou ils furent envoiés par le commandement de bouche à eux faict de Monseigneur le Gouverneur d'Orlians, pour savoir se lon pourvoit avoir aucune aide par devers le Roy Nostre Sire pour convertir en la fortification de la ville [...] Auxquels fut offert par les gens de Monseigneur le duc que, se ils voloient, ils avoient congié de apetisser leur mesure à vin, et plus non. Laquelle chose ils ne acceptèrent point ».

${ }^{8} \mathrm{CC}$ 538, fol. 2v: copie des lettres du roi Charles VI du 16 mars 1400 (n. st.), accordant «l'apetissement du dixième » de la pinte «sur toute maniere de gens, soit d'eglise ou autres », pour deux ans. Les lettres du 24 mars 1403 (n. st.) transforment l'apetissement en douzième pour trois ans (CC 539, fol. 6).

${ }^{9}$ Voir les ordonnances royales sur le guet. (Ord. t. VIII, p. 356-357, octobre 1399). 
consacrer trois quarts de l'aide obtenue du roi sur «l'appetissement» de la pinte, à la remise en état de la fortification et au perfectionnement de «l'emparement» de la ville $^{10}$. À ce subside, primitivement d'un dixième puis d'un douzième, on adjoignit une imposition sur les maisons et les rentes des "forins", contribution très mal acceptée par les intéressés ${ }^{11}$.

L'affectation propre des ressources entraîna deux comptabilités séparées, ordonnées cependant par le même receveur, visées par les mêmes procureurs et grossoyées par les mêmes notaires. Notre étude porte essentiellement sur les comptes de la forteresse, plus complets pour notre propos que ceux des « recettes et dépenses communes ». Le terme de forteresse ne doit pas nous induire en erreur, il ne s'agit pas seulement des tours, des murs, des ouvrages avancés, de l'armement, ce qui est la fortification matérielle de la ville. Un aspect plus subtil est aussi lisible dans ces comptes, celui des relations de la ville et de ses dirigeants avec un royaume en discorde et envahi par les Anglais ; aux yeux des procureurs, la diplomatie devient une forteresse, psychologique celle-là. C'est également de cette dernière qu'il va être question au cours de cette étude.

Il est évident que la majeure partie des deniers dépensés, mais il faudrait en établir l'exacte proportion, concerne les travaux de réfection et d'achèvement de l'enceinte, puis le renforcement des portes par des boulevards et le dégagement des murs par le nettoyage des fossés. Simultanément les travaux d'entretien nécessitent une surveillance et de menus ouvrages dont le compte rendu est assez répétitif. À partir de 1410, la ville se dote d'un matériel d'artillerie moderne, bombardes et canons supplantent les «engins» lanceurs de pierre, de type traditionnel. On entrepose traits, arbalètes et poudre au-dessus de la salle de réunion des procureurs, il y a là de quoi défendre une place dotée d'une garnison d'environ 200 hommes $^{12}$. La ville avait-elle les combattants pour se défendre ? Le problème se pose à chaque page des comptes à partir de 1407.

Des hommes, il y en a dans la ville. Des dizaines de travailleurs de tous les corps de métier : couvreurs, paveurs, forgerons, maçons, pionniers, voituriers, nau-

${ }^{10} \mathrm{CC} 539$, fol. 1v : «reddification, emparement de la muraille et des portes, pons et fossez et autres ». Clerc et laïques étant soumis, au moins en théorie, à l'aide; trois clercs commis par l'église souscrivent avec les procureurs à tous les mandements de dépenses et assistent à la clôture du compte.

${ }^{11} \mathrm{CC} 539$, fol. $7 \mathrm{v}$ : « subsides sur les maisons et rentes d'Orlians estant a gens forains et non demourans $[\ldots]$ ». CC 540, XXVII : procès avec l'abbaye de Bonneval (Eure-et-Loir), qui refusait de payer. CC 549, XXV : «A Jehan de Troyes [...] pour un mandement impétré devers le Roy $[. .$.$] à contraindre à la garde et à la fortification touz ceulx qui ont maison en la$ ville d'Orlians » (déc. 1426).

${ }^{12} \mathrm{P}$. Contamine, Guerre, État et Société à la fin du Moyen Âge, Paris-La Haye, 1972, p. 665, annexe XII, E : une note sur l'artillerie précise que, selon Christine de Pizan, il fallait pour une ville de 200 hommes de garnison, 54 arbalètes, 20 arcs, 37.200 flèches et traits, 2 bricoles, 2 couillards, 12 canons, 400 tampons, 1.000 à 1.500 livres de poudre et 3.000 livres de plomb. J'ai dénombré à Orléans, de 1410 à 1427, l'achat ou la confection de 47 arbalètes, 27.500 traits divers, 6 martinets à tendre les arbalètes, 96 frondes à bâton, 288 flèches et 144 cordes à arc (pas d'arcs...), 35 ou 37 canons avec des chambres supplémentaires, 2.000 pierres à canon, 1.600 livres de poudre, 600 «tappons». 
toniers, vanniers et surtout charpentiers ont œuvré des années durant pour dresser tout autour de la ville des défenses dignes d'une des plus importantes cités du royaume ${ }^{13}$. Quand les spécialistes ne sont point assez nombreux, on contraint les habitants, dûment enrôlés, à aller combler les fossés avec du «fient» contenu dans des hottes que par douzaines les vanniers vendent à la communauté, tandis qu'en d'autres endroits on sur creuse sous la responsabilité d'un sergent et de quelques notables ${ }^{14}$.

À parcourir les comptes entre 1415 et 1427 , on pourrait croire que les Orléanais ont passé leurs nuits à guetter et leurs journées à piocher. À côté de ceux dont c'est le métier, Bernard Josselin "guette» à Saint-Père-en-Pont et Simon Fournier son confrère à Saint-Pol ${ }^{15}$, il y a peu d'enthousiasme pour le guet, surtout aux périodes de crise, quand on double la garde et que 29 personnes chaque nuit veillent aux portes de la ville. Clercs et sergents passent des semaines entières, plusieurs fois par an, à asseoir le rôle de guet ${ }^{16}$. Le gouverneur, le prévôt et les procureurs doivent payer de leur personne pour donner l'exemple et galvaniser les cœurs prompts à l'affolement. En juin 1418, incertain du chemin que suivraient les Anglais, il fallut que, par deux fois, le gouverneur André Marchand fasse lui même le guet à cheval jusqu'à minuit «et le prevost, les procureurs et plusieurs autres le firent depuis minuit jusqu'au jour par dessus les murs ${ }^{17} »$. Charge exceptionnelle et commandée par un danger pressant, la fonction de garnison tenue par les bourgeois devient régulière quant à partir d'août 1419 trois hommes veillent à la Tour Neuve. On leur y installe une table, des sièges, deux lits dont les paillasses, les couettes et les couvertures sont régulièrement renouvelées ${ }^{18}$. Bien sûr, la ville avait organisé sa défense en se don-

\footnotetext{
${ }^{13}$ Cet aspect a fait l'objet d'une première étude d'ensemble : J. Leduc, Études sur les fortifications d'Orléans sous Charles VI et Charles VII, 1391-1431, d'après les comptes de forteresse de ta ville, D.E.S., mémoire principal sous la direction d'E. Perroy, Faculté des Lettres de Paris, 1966-1967 (dactylographié). Ce mémoire comporte en annexe une transcription du compte CC 545 (1415-1417).

${ }^{14}$ Le curage des fossés, commencé par des pionniers Denis Caro, Jean le Bourbonnais, Jean Lelong, en 1411 (CC 543, III), a rapidement nécessité l'aide des «bonnes gens» (ibid., XLVI). En juillet 1420, plus de 650 journées de travail ont été faites sous la responsabilité de 26 notables de la ville (CC 547, XLVIII). La liste des habitants devant travailler aux fossés a été dressée à la fin de 1412 ou au début de 1413 : «A Maistre Raoul du Hamel [...] avec Jehan Bruneau, sergent, pour mettre par escript ceulx qui devoient aller aux fossés « (CC 543, XXVI). CC 547, XVII : « rôle de ceulx qui vont recurer les fossez du matin au soir ».

${ }^{15}$ Apparus en 1409 (CC 542, IX) et assurant le guet "par jour et par nuict», tous deux sont encore là en 1427 (CC 549, XIX).

${ }^{16}$ Le guet apparaît le 22 novembre 1409 : le gouverneur était venu le 8 octobre à « dix heures de nuict dire que il falloit que l'on fist guet $»$. On l'organise en novembre (CC 542, II). Par la suite, le rôle est refait fréquemment (CC 543, II, IX, XIII, XX, XXVI ; 544, III, XVII, XXI ; 545, I, XVIII ; 546, XXXI, LVII, LXII; 547, XVII, XVIII, XXXI, LX ; 548, III, XIX, XXII ; 549 , V, XXI, XXIV etc.).

${ }^{17} \mathrm{CC} 546, \mathrm{LVII}$ : «pour meches à faloz prises le $\mathrm{VI}^{\mathrm{e}}$ de juin quand Monseigneur le Gouverneur et plusieurs bourgois d'Orlians et les sergens firent le guet à cheval jusqu'à minuit ».

${ }^{18} \mathrm{CC} 547, \mathrm{XV}$ : «A Jehan Godard, frippier, pour II coestes, II coessins de II liz mis en la tour Neuve le XXII aoust, II couvertures l'une de drap vermeil, l'autre une courtepoincte. [...] I
} 
nant en 1418 des cinquanteniers et des dizeniers, en leur fournissant des caisses de traits et en matérialisant les portions de muraille qui leur était affectées par l'inscription de leur nom sur le mur lui-même ${ }^{19}$. Mais ni la tour Neuve, ni le Châtelet ne sont à la charge de la ville, ils relèvent du duc et les hommes de celui-ci devraient en assurer le guet ${ }^{20}$.

Les bourgeois sont d'autant plus amers que les clercs, pas plus à Orléans qu'ailleurs, ne veulent assumer les charges de la défense. En 1418, il fallut saisir les rentes des gens d'église pour obtenir une participation à la taille supplémentaire pour la forteresse ; les procureurs ont également noté la jurisprudence royale en matière de guet: ils ont fait faire copie d'un «appoinctement» du Parlement touchant à un conflit entre la ville et l'église de Saint-Riquier «pourquoi iceulx religieux se vouloyent exempter du guet ${ }^{21} »$. Quant à l'Université (et ses «suppôts»), hostile aux charges civiles par principe, elle accumule les vidimus confirmant l'exemption de guet de ses officiers sinon quand l'ennemi est à moins de dix lieues, nisi inimici capitales fuerunt prope villam Aurelianensem de decem leucis (1411), ou même tempore guerrarum (1428). La ville riposte en obtenant de Charles, roi de France, des lettres patentes rappelant que tous les habitants d'Orléans sont tenus au service de guet, de garde et aux impôts de fortification ${ }^{22}$.

Le guet permet de ne pas être surpris au dernier moment, de fermer à temps les portes, de prévenir au son des cloches les arbalétriers et les gens qui ont des "harnoys » et que l'on a soigneusement répertoriés, de se porter aux murs et aux portes $^{23}$. Même si on peut espérer ne pas être surpris, il n'en faut pas moins savoir sur qui compter quand surviendra le danger. Un des soucis des responsables de la forteresse consiste à essayer de tisser autour de la ville tout un réseau de «sûretés" qui, ajoutées à l'aspect formidable de la fortification pourrait dévier les menaces. Si soucieux qu'ils soient de leurs prérogatives urbaines, les bourgeois d'Orléans savent qu'ils ne peuvent et ne doivent assumer entièrement leur sécurité : ce n'est pas leur rôle dans la société. La ville a donné son nom à un duché, à un duc, tout un ensemble de liens très forts unit la cité à son seigneur, et au pays d'Orléanais. Lorsque rien ne menace, l'éloignement du duc de sa capitale et la non-résidence de son gouverneur sont très bien admis : on les reçoit en visite, on les nourrit, on les abreuve, on

charette de pailhe pour les II liz [...] II chasliz touz neufs pour les II liz ou couchent chascun jour trois des habitans de la ville».

${ }^{19} \mathrm{CC} 546$, V. Le nom des cinquanteniers nous est donné au mandement XX : Robin de SaintMesmin, Jean Aubelin, Jacquet Labbaye, Guion Du Fossé, Huguet Morinet, Jean Mignon.

${ }^{20} \mathrm{R}$ Boucher de Molandon, «La Tour Neuve», Mem. Soc. Archéol. et Hist. de l'Orléanais, t. XVIII, 1884, p. 89-101.

${ }^{21} \mathrm{CC} 546$, III.

${ }^{22}$ M.-H. Julien de Pommerol, Sources de l'Histoire des Universités françaises au Moyen Âge, Paris, I.R.H.T., 1978, p. 83 (1411), p. 196 (1427 et 1431), p. 426 (1428).

${ }^{23}$ Les comptes mentionnent plusieurs dons faits aux arbalétriers pour l'entretien de leurs buttes (CC 545 (1416), VII), comme pour en construire quand l'avancée des boulevards mord sur leur terrain, également pour leur édifier une «loge» où se retirer en cas de pluie (CC 547 (1420) XXVIII). En 1417-1418, on s'inquiète de l'armement des bourgeois : visite de « ceulx qui estoient armez, et aussi les contraindre à iceulx armer et faire inventoire de leurs harnoys » (CC 546, VII). 
leur envoie du poisson de Loire et des étangs de Sologne dans de vastes draps ou de grands bassins. En bienvenue, la ville consacre le tiers de sa recette en vaisselle d'argent pour Louis en $1392^{24}$. Ce type de relation n'a d'ailleurs rien que de très normal, toutes les villes procèdent de même. En ces années fastes, on peut se permettre d'avoir quelques différends avec l'administration ducale à propos des panonceaux qui décorent la muraille. La ville a ses propres armes, elle peut s'en orner, les faire figurer à côté de celles de son seigneur ou de la duchesse, comme en 1393 pour la visite de Valentine Visconti ${ }^{25}$. Ce sont des ornements provisoires, bien différents des panonceaux qui, de façon permanente somment les tours ou encadrent les portes, et dont le langage n'est pas neutre. Lors de la reconstruction des tours et des salles des portes, on scellait au sommet des couvertures un pommeau de plomb auquel était attaché un panonceau de fer peint. Il en fut ainsi aux lucarnes de la Porte Bernier $^{26}$, au faite de la tour Au Villain et de la tour de l'Evêque, au sommet des deux tours de la Porte Bourgogne ${ }^{27}$. Parfois, la décoration était plus poussée : le Portereau (les Tourelles) avait une image sculptée de Saint Jacques, sous un «tabernacle» repeint à neuf; la tour Micheau Quanteau était surmontée d'un Saint Michel d'airain ${ }^{28}$. La nouvelle entrée de la Porte Bourgogne arborait sur sa grande lucarne trois anges tenant l'écu du duc, le tout «d'or fin et de bon azur»». Mais la ville sur cette porte payée de ses bons deniers osa mettre deux écus à ses armes ${ }^{29}$. Colère du gouverneur, Pierre de Mornay, qui veut les faire abattre. Girard Groussard, le receveur responsable de la construction, vint à Paris et porta «la dicte porte toute pourtraicturee [en deux exemplaires] afin que l'on vist l'ordonnance des deux escus, laquelle pourtraicture il montra oudit chancellier, lequel luy dict que c'estoit tres bien faict et se merveilloit moult dont le dict gouverneur les vouloit faire oster, et manda oudit gouverneur qu'il savoit bien que les armes des bonnes villes de France estoient aux portes audessoubs des armes du Roy nostre sire et des autres seigneurs » ${ }^{30}$. Par la suite, les panonceaux aux armes du duc continuèrent à briller au soleil sur les tours couvertes d'ardoises, à la Porte Chesneau, à la tour "près la tour Neuve » (tour Blanche actuelle), sur les huit lucarnes du Portereau et les deux célèbres Tou-

${ }^{24} \mathrm{CC}$ 537, fol. 31v: Vaisselle d'argent pour le duc, payée 1330 francs, alors que la recette totale de la ville (il n'y avait qu'une comptabilité) s'élèvait à 32461.15 s. 6 d.p.

${ }^{25} \mathrm{CC} 537$, fol. 33 : confection de 7 écus pour les portes, de 48 petits aux armes de la duchesse et de la ville, d'autres encore pour les torches.

${ }^{26} \mathrm{CC} 537$, VII : peinture des panonceaux; ibid., $\mathrm{X}$ : fourniture des panonceaux de la porte Bernier ( = Bannier).

${ }^{27} \mathrm{CC} 539, \mathrm{XXX}$ : Tour Au Vilain (seule lecture possible du nom de cette tour, appelée tour de Messire Baudes ; (CC 546, LXVI) à partir de 1419), peinture aux armes du duc ; CC 540, XVII : à Etienne le Normand, peinture du panonceau de la tour «à l'encontre de l'ostel de Révérend père en Dieu Monseigneur l'Evesque d'Orlians »; CC 539, XLVII : Etienne le Normand, 3 panonceaux de la Porte de Bourgogne.

${ }^{28}$ CC 539, XXXVIII : «A Bernard Vincent, pour une ymage de pierre de Monseigneur Saint Jacques pour mettre à ha porte du pont, et sera icellui ymage de III pieds et demy de hault [...] LXIII s.p.»; ibid., XLVII : « un bassin d'airain duquel len a faict l'ymage Saint Michel qui est sur la tour Micheau Quanteau ».

${ }^{29}$ CC 539, XLVII.

${ }^{30} \mathrm{CC} 540, \mathrm{X}$. 
relles $^{31}$. Mais la ville hissa ses propres armes sur ses tours de guet, visibles de loin, peintes sur les deux faces de grandes bannières de toile ${ }^{32}$. Et quand le duc fut prisonnier et que le traité de Troyes fut près d'être juré, les procureurs firent mettre pour "l'honneur et la seureté » de la ville des lances et deux «pavas » à ses armes aux cinq portes $^{33}$. La ville craignait en effet de ne devoir plus guère compter que sur ellemême.

Avec des crises et des rémissions, les dangers se précisent. Tout au long de ces décennies, les comptes rendent parfaitement sensibles les efforts des dirigeants de la ville pour essayer de dominer les vagues de panique qui s'étendent à travers le pays à l'approche de toute troupe d'hommes d'armes.

Avant tout, savoir. Le danger, c'est une nuit avec, à ses portes, des étrangers indésirables, ou des amis encombrants ; c'est la présence continue de compagnies dans les campagnes. Mais c'est également passer à côté des alliances en train de se nouer, des trêves en gestation; c'est de ne pas être recommandé à un puissant du jour, de ne pas avoir su choisir son camp.

Le service de renseignement fait partie des charges de la forteresse, selon les conceptions des procureurs. Certes, ils ne peuvent payer en permanence des chevaucheurs parcourant les campagnes, sinon pendant le dur passage de $1418^{34}$. Cependant, lors de toutes les crises, des gens reçoivent des gratifications pour rapporter à Orléans les avances et mouvements des troupes. Pendant la conspiration de 1412, un homme part pour Bourges «savoir des nouvelles et en rapporter» (10-15 juin), puis un chevaucheur du duc porte des lettres faisant «mention comment l'accord avoit este faict à Ausseure entre Messeigneurs de France ${ }^{35} »$. On se préoccupe des mouvements des Bourguignons en 1416 et en septembre $1417^{36}$. Mais surtout on redoute les Anglais. En septembre 1412, pendant la chevauchée du duc de Clarence, une grande agitation règne pour savoir où passeront les colonnes ennemies; hélas celle de Messire «Jehan Blunt» (John Blount) passe par Orléans au grand dam des procureurs qui durent racheter d'une aiguière d'argent et ses six gobelets portés au capitaine anglais « un prisonnier que menoient ses gens, auquel prisonnier le filz au Lièvre coppa la corde au Portereau et le fist eschapper [...] dont ledit chevalier menaça prendre des bourgois [...] et faire plusieurs grands maul $x^{37} »$. Une seconde vague de

${ }^{31} \mathrm{CC} 542, \mathrm{XXVII}:$ Étienne le Normand pour 6 panonceaux (1410).

${ }^{32} \mathrm{CC} 546, \mathrm{~V}$ : une toile «ardent» pour la bannière de Saint-Paul, frais de la peinture par Étienne « le Paintre».

${ }^{33} \mathrm{CC} 547$, XVIII et XXX (juin 1420).

${ }^{34} \mathrm{CC} 546, \mathrm{XXXII}$ : «au Prevost d'Orlians pour avoir touz les jours des chevaucheurs » (fév. 1418 n.st.). CC 546, XXXVI : même chose, mai 1418.

${ }^{35} \mathrm{CC} 543, \mathrm{~V}$ : Voyage de Perrin Gerbaut à Bourges. Ibid., VI : Jean Salmon, chevaucheur du duc. Il s'agit de la paix d'Auxerre, 22 août 1412.

${ }^{36} \mathrm{CC} 545, \mathrm{X}$ : «A Jehan Salmon, chevaucheur de Monseigneur [...] pour savoir quel chemin vouldroient prendre une grand assemblée de Bourgoignons et de bannis qui estoient venus près de Berry, et disoit lon qu'ilz vouloyaient venir assaillir Lorriz en Gastinois ». (Mandement du 30 mars 1416 n.st.). CC 546, V : voyage de Jean Hareng, sergent du duc pour voir si les Bourguignons n'étaient pas vers Etampes (sept. 1417).

${ }^{37} \mathrm{CC}$ 543, VIII : 14 septembre 1412, les procureurs décident de demander à Blois «se les Angloys estoient environ Bloys, ne en quel lieu ilz estoient ». Ibid. : voyage pour savoir au- 
panique naît avec le débarquement d'Henry V; en septembre 1417, des espions partent vers Falaise et Caen savoir « quel chemin le roy d'Angleterre vouldroit prendre». En février suivant, le bruit court que les armées anglaises sont à Brou en Beauce ; on paie alors une (belle ?) espionne normande pour aller "visiter» ces Anglais, lesquels la détroussent de son manteau et la soulagent de sa bourse, mais lui permettent de retourner à Orléans où, ayant rempli vaillamment sa mission, elle rapporta que les ennemis «faisoient grant amas de charroy» tout en retournant vers Verneuil ${ }^{38}$. D'autres messagers sont envoyés en Normandie, sur la Loire par bateau, et par «touz le pais». Ils reviennent avec de mauvaises nouvelles : les Anglais sont à Authon-du-Perche, ils ont saisi deux épiciers, ou de meilleures: Messire Robert Poulet, chanoine, est allé jusqu'au Bec en mai 1418, il revint en disant que «le dict Roy (d'Angleterre) vouloit aller droit a Rouen", ce qui détourna les inquiétudes orléanaises $^{39}$. Elles renaissent l'année suivante et les édiles trouvent un frère carme, Jean Belheure, prêt à monnayer, à l'instar de nombreux Mendiants, les renseignements sur «l'estat des Angloys" glanés au cours de ses pérégrinations caritatives vers Dreux ou Bonneval ${ }^{40}$. La crainte des Anglais entraîne l'accélération des travaux des boulevards en avant des portes nord et ouest, les plus exposées ${ }^{41}$. Cette inquiétude fait naître une vague de xénophobie ; déjà en 1412, les sergents s'étaient déplacés auprès des hôteliers afin d'obtenir la déclaration de ceux qu'ils logeaient ${ }^{42}$. Ils refirent les mêmes démarches en octobre 1418 et en juin 1419; étranger ne signifiant pas seulement Anglais, les 17, 18 et 19 juin 1418 le prévôt en personne alla «visiter les escoliers estrangers ${ }^{43} »$.

Les armées anglaises sont redoutables, les amis ne valent guère mieux ; quant à ceux dont on ne sait qui ils sont... Alors deviennent choses quotidiennes les troupes qui battent la campagne, surgissent au moment des récoltes, se font payer leur recul avec du vin.

Nous ne saurions énumérer exhaustivement le nombre des compagnies qui passèrent par la ville ou par son "pays », depuis les hommes partant avec le duc Louis en Guyenne en 1406 et qui burent force vin à Saint-Jean-de-Braye ${ }^{44}$, jusqu'aux troupes de la malheureuse journée des harengs de février 1429. Il y eut entre autres «les Picars» traînant en Beauce en $1409^{45}$, puis les troupes au retour de Bourges,

près de ceux de Blois «quel don ilz avoient faict aux Angloys »; Ibid., IX : 23 septembre, poissons présentés à Messire Jehan Blunt, chevalier d'Angleterre «lequel estoit venu à Orlians pour veoir la dicte ville ». Ibid., affaire du prisonnier.

${ }^{38} \mathrm{CC} 546$, XXXIX : il s'agissait de la chambrière de «Frère Jehan de Bourges, religieux de Boigny ».

${ }^{39}$ Ibid., LVI : mandement du 19 mai 1418.

${ }^{40} \mathrm{CC} 547$, IV et X (1419).

${ }^{41} \mathrm{CC} 546, \mathrm{XXII}$ : visite des procureurs et du gouverneur autour de la ville pour voir « où l'on feroit les boulouars » (1418)

${ }^{42}$ CC 543, VIII : Jean Cazeau, notaire, et Jean Myot, sergent, «pour avoir esté dans toutes les hostelleries d'Orlians [...] dire que se il y a un estranger logé, l'hostellier le fasse dire à la justice ».

${ }^{43}$ CC 546, LX

${ }^{44} \mathrm{CC} 541$, fol. 14 et $23 \mathrm{v}$.

${ }^{45}$ CC 542, XVII : «Vuider les Picars logiés à Saint Lop d'Ingré ». 
l'été de $1412^{46}$. Olivier de Breban et Armenon d'Albret tinrent la campagne tout l'hiver de $1413^{47}$, suivis par les capitaines qui rentraient de l'ost d'Arras en septembre $1414^{48}$. Si les hommes du comte d'Armagnac furent en partie détournés à la fin de $1415^{49}$, ceux du comte de Tonnerre campèrent l'hiver de $1416^{50}$, précédant le passage des arbalétriers génois en août $1417^{51}$, auxquels succédèrent les gens revenant de Falaise à l'automne de la même année ${ }^{52}$. En 1418-1419, c'est le reflux des Armagnacs de Paris vers Touraine et Poitou, et l'arrivée de renforts du Languedoc ${ }^{53}$. De 1419 à 1429, partisans du Régent et incursions anglaises mettent à rude épreuve les nerfs des procureurs : sur les talons de J. de Buchan se présentent les Anglais en décembre $1421^{54}$. Il faut insister auprès du Régent pour écarter les ravages des hommes du vicomte de Narbonne au cours de l'été $1422^{55}$. Aux exactions du sire de Galobie autour de Janville en $1425^{56}$, fait écho la terreur des bords de Loire quand

${ }^{46} \mathrm{CC} 543$, VI : dépenses faites en commun avec les gens de Blois au retour de Bourges "quand le siège fust levé ».

${ }^{47} \mathrm{CC}$ 544, III à IX : hommes d'armes d'Olivier de Breban, de M. de Sainte Mesme. Ibid., XIX : « au bastard de Riez pour sa peine d'avoir esté a Noan et Mer [...] a Armenon Dalebret et Poinçon de la Tour pour leur prier comment ilz ne passassent pas par Orlians ».

${ }^{48} \mathrm{Ibid}$., XXIII : «faire vuider les gens d'armes à la revenue de l'ost du Roy Nostre Sire de devant Arraz et pour garder le pais de Monseigneur d'Orlians ». Je suppose qu'il faut lire que ce sont les gens qui reviennent d'Arras qui «vuideront» ceux qui pillent l'Orléanais.

${ }^{49} \mathrm{CC} 545$, IV : les gens d'Orléans obtiennent du comte d'Armagnac des lettres destinées à Jean de Broquiers, pour lui demander de ne pas passer par Orléans. Ils suggèrent le passage de la Loire par Jargeau !

${ }^{50} \mathrm{Ibid}$., XVII : voyages de Jean Aubelin, procureur, à Jargeau «pource que les gens au comte de Tonnerre $[. .$.$] venoient au pais devers Orlians ».$

${ }^{51} \mathrm{CC}$ 546, VII : dons (poissons, perdreaux, pigeons) portés au sire de Castelle, capitaine des arbalétriers de Gênes pour «le supplier qu'il deffende à ses gens qu'ilz fassent le moins de mal qu'ilz pourroient entour Orlians » (5 août 1417).

${ }^{52} I b i d$., XXII : don de vin pour les capitaines de gens d'armes «qui venoient de Falaise et estoient logés a Orlians ».

${ }^{53} \mathrm{Ibid}$., LXII : 22 août 1419, le prévôt et les procureurs d'Orléans font passer les gens du prévôt de Paris par Saint-Jean-de-Braye ; ibid., LXIV : les procureurs envoient une lettre close à Monseigneur de Vertus (le frère de Charles d'Orléans) à Blois «qu'il lui plusse contre mander les gens d'armes qui estoient vers le Bourgdedieux affin qu'ilz ne vinsissent point par deçà, pour les vendanges qui se approssoient » (sept. 1419 - Bourg de Dieu : Déols, Indre).

${ }^{54}$ CC 547, XXXIII : 21-23 juillet 1420, volailles pour Monseigneur le comte de Bouquan (Jean Stuart, comte de Buchan, connétable de France de 1420 à 1424) et pour le connétable d'Ecosse (Jean Stuart, seigneur de Darnley) : 6 chapons de «haulte gresse », 4 douzaines de poussins, 4 douzaines de pigeons, 3 lièvres, 192 pintes de vin, pour 6 repas. CC 548, III : demande au Régent «par le conseil de Monseigneur le Chancelier d'Orlians... que les gens d'armes de mondict seigneur se reculassent affin que l'en pust cueillir l'aoust »; ibid., XI : mandement du 3 février 1422 n. st., "pour faire boire les compaignons qui estoient armés au Pont le jour que les Anglois furent devant ladicte ville».

${ }^{55} I b i d .$, XXV : voyage des procureurs à Blois pour « exposer les maulx que les gens de Monseigneur de Narbonne font au pays de Monseigneur».

${ }^{56} \mathrm{CC} 549$, VIII : voyage du Héraut Croissant à Poitiers à cause « des griefs que font un chascun jour les gens de Galobie [...] comme de tuer gens, avoir assailli Janville, avoir ars les molins à vent et les charrues es champs » (automne 1425). 
surgirent en août 1425 les compagnons de Jean de Grailly ${ }^{57}$. Ce fut bien pis quand Pithiviers fut prise et que, de juillet à septembre 1427, capitaines du roi et hérauts des Anglais se présentent alternativement aux portes de la ville. On n'a qu'un écho discret du sauvetage de Montargis par le bâtard d'Orléans ${ }^{58}$.

Dans ces conditions s'expliquent encore mieux la fébrilité des travaux et la demande de subsides exceptionnels (mais qui deviennent réguliers), quand le Régent Charles s'installa en Berry. Il octroya en 1421 un subside sur les «danrees et vivres yssans hors de la ville» et une «crue » sur le sel vendu aux greniers à sel d'Orléans, le duc, prisonnier, recevant un tiers de cette crue ${ }^{59}$. On comprend également la commande à Naudin Bouchard, entre deux bombardes, de quatre cloches «a mettre sur les murs de la dicte ville, a esveiller la garde et [mises] sur les portes qui enssuivent et pesans, c'est assavoir celle du Pont CVII 1., celle de la Porte Bernier LXVI 1., celle Porte Saint Aignan LXXVIII 1., celle Porte Parisie LV 1.», cloches s'ajoutant aus «saincs » de Saint-Paul et de Saint-Père en Pont ${ }^{60}$.

La protection des remparts, du guet, de six tocsins, est peut-être suffisante pour faire face à une surprise, elle ne l'est pas pour assumer les répercussions des choix politiques de la ville. Ce choix d'ailleurs n'est qu'en partie le sien, il lui fut dicté, comme celui de Blois avec laquelle elle ne cessa de se consulter, par l'appartenance à l'apanage de Charles ${ }^{61}$. On ne pouvait être Bourguignon... Le choix du parti du Dauphin fut dicté par les princes d'Orléans, Charles, depuis sa résidence forcée, et Philippe de Vertus, frère du duc, qui convoqua les procureurs le 11juin 1418 : il leur annonça l'accord, passé à Gien, avec le Dauphin, et obtint d'eux une

${ }^{57} \mathrm{CC} 549, \mathrm{XXV}$ : «à Guillaume Simon et Jehan Hate qui ont estés ordonnez a mettre gens sur les portes, non obstant les guetes, tant que le comte de Foix fut par deçà... l'espace de XLV jours $»$.

${ }^{58} \mathrm{CC} 653$ (compte de ville, 1427-1429), IX : voyage à Jargeau pour savoir des nouvelles de Montargis et don de vin à un héraut anglais; ibid., X: chapons au connétable de France ; ibid., XXIV : vin envoyé au comte de Salisbury par l'intermédiaire de deux hérauts anglais venus de Janville.

${ }^{59} \mathrm{CC} 548$, fol. 6v : «recette de subsides mis de par le Roy Nostre Sire, pour lors Régent le Roy, sur certaines danrées et vivres yssans de la ville». le subside portait sur les draps, literie, friperie, sur les cuirs, sur les harengs et poissons salés, le bétail ; ibid., fol. $7:$ «recette d'une crue de III 1. XVI s.p. sur chascun muy de sel à la mesure de Paris, vendu es greniers à sel en la dicte ville d'Orlians [...] en laquelle crue Monseigneur d'Orlians prend la tierce partie ».

${ }^{60} \mathrm{CC}$ 547, LVII, LVIII: la cloche du pont était sur un «campannier [...] près de Saint Anthoine, sur le ponts », érigé entre le 8 et le 15 février 1421, les autres furent suspendues à des beffrois de charpenterie..

${ }^{61} \mathrm{Cf}$. note 37. Lors des tractations pour l'abstinence de guerre, en 1424-1426, Orléanais, Blésois et Vendômois se sont unis pour financer la mission de La Trémoille : «à Monseigneur de La Trémoille et de Suli auquel les habitans de la dite ville et du duché d'Orlians et des contez de Blois et de Dunoys, assemblez audit lieu d'Orlians, ont octroyé la somme de IIm escus d'or pour poursuivre certaine abstinance de guerre par devers le duc de Bourgoigne, porcionnée ladite somme, c'est assavoir pour ladicte ville d'Orlians, XIc LXVI escus et II tiers d'escu, et pour les dits comtez. VIIIc XXXIII escus I tiers ». La solidarité entre les villes prenait parfois un aspect « sportif » : « à Jacquet Compaing, arbalestrier d'Orlians, pour aller à Blois au secours des compaignons arbalestriers de Blois lesquels les avoient mandés contre les arbalestriers de Tours qui avoient deffait ceulX de Blois à jouer» (CC 545, XX, 1417). 
«réponse» dit pudiquement la note de frais ${ }^{62}$. Cependant, la politique de la ville est fort prudente et les pintes de vin sont versées généreusement, tant et si bien qu'en octobre 1420 trois procureurs durent aller se disculper et dénoncer «ceulx qui avoient accusé la dicte ville et publié certaines paroles audict Monseigneur le Régent, que les manants et habitans de la dicte ville avoient faicte alience avec le roy d'Angleterre, desquelles choses il ne fust oncques rien trouvé». Ils obtinrent des lettres pour "savoir et enquérir d'où estoient venues les mauvaises parolles ${ }^{63}$ ». À dire vrai, les registres étaient rédigés dans les deux années qui suivaient l'exercice, il peut y avoir eu une certaine édulcoration. Cependant, même s'il y eut quelques faiblesses, une preuve assez amusante de l'opposition aux Bourguignons est donnée par les baptêmes successifs de la porte orientale de la ville. C'était la porte de Bourgogne... Subitement, au folio 30 du compte de 1409-1411, soit à l'automne de 1411, et pour marquer la réprobation orléanaise à la rentrée des assassins de son duc à Paris le 22 octobre, la porte devint «Saint-Aignan» du nom de l'abbaye toute proche. Ce nom lui est donné jusqu'en 1417, quand pour quelques mois elle redevint Bourgogne. Saint-Aignan à nouveau à partir de mai $1418^{64}$, elle le reste jusqu'au siège, pendant lequel on sait que les Orléanais ont demandé à Philippe Le Bon de les aider, ce qui a provoqué sans doute le retour au nom primitif, et c'est bien par la porte de «Bourgogne» que Jeanne d'Arc fit son entrée le 29 avril 1429.

Débaptiser une porte ne demande pas de démonstration de force; mais qui commandera à ceux qui jouent des bombardes, qui viendra poursuivre et «riser» les gens d'armes qui ravagent les faubourgs? Tant qu'il y eut un duc, qu'il soit à Paris ou à Blois, la situation était simple, les procureurs allaient voir son gouverneur, ou son chancelier ou le prince lui-même. Les premières années, alors que rien de bien sérieux ne menace, le gouverneur, Jean de Prunelle (ou Prunelé), qui exerça ses fonctions de 1391 au 13 septembre 1404, n'apparaît que peu dans les comptes sinon pour ses gages. Souvent les procureurs font l'économie d'un voyage auprès de lui et vont directement vers le duc. En 1404, Pierre de Mornay dit Gaulnet, seigneur de la Ferté-Nabert (la Ferté-Saint-Aubin), est gouverneur ; c'est lui qui aura à faire face aux problèmes posés par la mort de Louis et la captivité de ses deux fils, Jean, comte d'Angoulême, en novembre 1412, Charles à Azincourt en octobre 1415. Les officiers des princes, Pierre de Mornay, Jean de Gaules ${ }^{65}$ et Matho de Cheveaulx, un simple chevalier solognot, se sont dépensés sans compter pour la ville d'Orléans de 1407 à 1420-1421; c'est là un des aspects les plus intéressants des comptes de la cité.

Avec Philippe, comte de Vertus (1396-1420), second fils de Louis d'Orléans, ces hommes ont réussi à maintenir la ville à peu près à l'abri des gens d'armes pen-

${ }^{62}$ CC 546, LVIII : retour des procureurs de Jargeau où ils avaient parlé «à Monseigneur de Vertus qui les avoit mandés pour leur dire ce qu'il avoit faict à Gien par devers Monseigneur le Daulphin» (11 juin 1418). Voyage de P. Compaing, Berthier, Renart, Berthaud Mignon, Raoulet du Recourt, avec Maître Guillaume Gebel et Jean du Refuge, le 21juin, pour donner la réponse de l'église et de la ville (ibid., LXI).

${ }^{63} \mathrm{CC} 547, \mathrm{~L}$ (15-20 octobre 1420) : un bruit semblable avait couru en 1410 sur des livraisons par la ville de blé aux Anglais (CC 647, XVI).

${ }^{64} \mathrm{CC} 546$, II à XXIV : Porte Bourgogne ; LVII et suivants : Porte Saint-Aignan.

${ }^{65}$ L. Monnier, Notice sur Jehan de Gaules (dactylographié), Archives du Loiret, 1961. 
dant les périodes les plus sombres. Dès qu'une menace se précise, les bourgeois se précipitent vers «Monseigneur », de Gaules ou le gouverneur. En réponse, outre de bonnes paroles, le comte se déplace ${ }^{66}$, le gouverneur vient faire le guet en $1409^{67}$, ou délègue Matho de Cheveaulx pour apaiser les esprits et redonner du courage, traiter avec les capitaines ${ }^{68}$. J. de Gaules voulant partir en 1416 en Guyenne, convoque les procureurs pour leur dire qu'il n'oublie pas la ville ${ }^{69}$. Il est certain que tant qu'un membre légitime de la famille ducale a pu assumer la charge du duché, la ville n'a cherché qu'en lui le protecteur dont elle avait besoin. Philippe de Vertus est venu « au giste » plusieurs fois, il a visité la forteresse, c'est lui qui a fourni, dans les forêts ducales, le bois des boulevards ${ }^{70}$. C'est lui qui conseille aux dirigeants d'Orléans d'aller ou non se recommander au Dauphin ${ }^{71}$; en novembre 1418, il sert de médiateur quand la ville renâcle à fournir les hommes demandés ${ }^{72}$. Il ne cesse d'intervenir contre les compagnies. Aussi sa mort fut-elle ressentie comme un désastre, et alors que les comptes de forteresse ne mentionnent ni la mort du duc Louis ni le désastre d'Azincourt (ce que font les comptes de ville ${ }^{73}$ ), et ne parlent du comte d'Angoulême que parce qu'il fallut payer pour sa rançon, ils enregistrent le désarroi des procureurs en septembre 1420: «A Maitre Jehan Noaille, docteur, Maitre Amy Gombert, Maitre Jehan Davy, Estienne Germe, Guiot Boileve, Thevenin de Bourges et Jehan Aubelin [...] pour recommander la ville et le pays à Monseigneur le Régent pource que Monseigneur de Vertus est allé de vie a trespassement » ${ }^{74}$.

${ }^{66} \mathrm{CC}$ 544, V ; 546, XIX, Philippe arrive de nuit de Paris (1418) ; ibid., XXVIII, il visite la forteresse.

${ }^{67} \mathrm{CC} 544$, II : cf. note 16.

${ }^{68} \mathrm{CC} 544, \mathrm{XX}$ : en 1414, les procureurs envoient Matho de Cheveaulx « au devant des gens d'armes qui estoient à Jehan des Hayes pour les faire passer par Blois, et n'y passerent pas mes passerent par Beaugency, et les convoya ledit Matho jusque à Varize » (Varize : Eure-etLoir, canton : Orgères-en-Beauce).

${ }^{69} \mathrm{CC} 545$, IX : «il avoit ordonné certaines gens pour le gouvernement de la ville... pource qu'il voloit aller en Guienne avec Monseigneur de Sully ».

${ }^{70} \mathrm{CC} 546, \mathrm{XXXII}$ : paiement au secrétaire du Duc de «la lettre du don du boys que Monseigneur de Vertuz a donné à la ville pour faire les boulouars » (mandement de févier 1418 n.st.).

${ }^{71}$ CC 546, LXVI : 11 novembre 1418, voyage de deux procureurs et d'un homme d'église à Meung «par devers Monseigneur de Vertuz pour avoir son conseil se il seroit bon que on alast à Jargeau par devant Monseigneur le Daulphin pour lui recommander la ville et le pays ».

${ }_{72}$ Ibid., LXXXII : «à Pierre Renier, tresorier de Monseigneur le duc d'Orlians, pour argent a lui baillé pour une composition faicte a Monseigneur le Chancelier d'Orlians et aux gens du Conseil de mondit Seigneur, pour Monseigneur le Daulphin, Regent le Roy, qui avoit escrip par deça que la ville lui envoyast XX hommes d'armes et XII hommes de traict payés pour II mois pour tenir sur les champs avec d'autres hommes d'armes qu'il mettoit sus [...] De laquelle composition fut chargié Monseigneur de Vertuz ».

${ }^{73} \mathrm{CC} 646, \mathrm{XV}$, compte de ville (1407) : paiement des écus peints pour l'église Saint-Samson pour le jour des obsèques de Louis d'Orléans. CC 649, VIII : 27 octobre 1415, voyage «pour aler savoir la vérité de la journée, comment il en avoit esté des gens du Roy nostre sire, à l'encontre de son adversaire le Roy d'Angleterre ».

${ }^{74} \mathrm{CC} 547$, XLI. 
Il fut aidé par des chevaliers du duché comme Matho de Cheveaulx, resté par ailleurs fort obscur, mais qui a passé au service de la ville, en tant que Lieutenant du Capitaine des journées et des nuits à cheval pour assumer la délicate mission de reconduire les gens d'armes hors du pays. Sur la brèche par deux fois en $1409^{75}$, il multiplie les missions de 1413 à 1420, fait le guet, convoie les indésirables au-delà de Beaugency ou de Jargeau; trois jours durant, il discute avec les procureurs, en septembre 1414, de la levée d'une taille de 800 francs "octroyee à Messire de Chaumont, avec luy cinquante lances pour faire vuider les gens d'armes... et pour garder le pays de Monseigneur d'Orlians... », taille proposée par le duc et qui sera finalement éludée. Matho règle la question en un premier temps en envoyant aux soldats de l'avoine, du pain et de la chandelle... puis Bouchard de Mornay, frère du gouverneur, les conduit «oultre Chier» dans le duché voisin" ${ }^{76}$ ! En 1417, Matho tient garnison à la tour Neuve ${ }^{77}$. Quand en 1418, on voulut donner, sans doute à l'initiative du nouveau gouverneur André Marchand, une garnison à la forteresse lors de l'avancée des Anglais, la ville s'affola et envoya deux procureurs auprès du comte de Vertus "pour luy dire que l'on nous vouloit bailler garnison de gens d'armes, dont monseigneur de Vertuz nous envoya Matho de Cheveaux ", au grand soulagement de tous. La ville le garda du 22 mars au 22 août gagé à 30 s.p. (18 payés par la ville et 12 par le gouverneur $)^{78}$. Nous avons là un des exemples de l'attitude de la petite noblesse locale, consciente des charges de son rang, assez éloignée des critiques à elle adressées par le Quadriloge invectif.

L'arrivée des hommes du Dauphin et la mort du comte de Vertus inaugurent une nouvelle phase. Après une arrivée assez fracassante (changement immédiat de toutes les serrures de la ville, creusement des fossés, multiplication des voyages), le gouverneur André Marchand sur place depuis 1418 se révèle beaucoup moins efficace diplomatiquement que son prédécesseur ; n'ayant plus l'appui d'un prince proche du duc Charles, il fut vite débordé par l'entourage du Régent. À peine le comte enterré, celui-ci propose la nomination du seigneur de Mortemar comme capitaine de la forteresse, ce qui ne se fit pas $^{79}$. Une dernière fois, Pierre de Mornay et Matho

${ }^{75} \mathrm{CC} 542$, fol. $12 \mathrm{v}$ : «A Matho de Cheveaulx, lieutenant du capitaine pour aller aux Bordes Allais en Beausse vuider les gens d'armes qui y estoient... y fust avec luy le Grand Colas, sergent, et ce fust le XIIII ${ }^{\mathrm{e}}$ 'aoust [1409]»; ibid., fol. 20.

${ }^{76}$ CC 544, XXIII : réunion des $17,18,19$ septembre 1414 ; ibid., XXVII : convocation de 80 bourgeois pour discuter; ibid., XXVIII : Matho fait distribuer avoine, pain (2 douzaines), chandelle.

${ }^{77} \mathrm{CC}$ 546, XXI : Matho surveille l'installation des bombardes de la ville, il est là le jour où passent les gens d'armes du comte de Penthièvre; id., XXIII : poisson pour les gens de la tour Neuve, une table de 9 pieds de long et 2 tréteaux; ibid., XXVIII : le jour de la Sainte Catherine, 2 pots de vin pour la femme de Matho de Cheveaulx à la tour Neuve.

${ }^{78} \mathrm{CC} 547$, IX.

${ }^{79} \mathrm{CC} 547, \mathrm{XLV}$ : «A Etienne Germe, procureur général de ladite ville, pour un voyage a Meung sur Yevre... faisans mention comment ilz avoient esleu Monseigneur de Mortemar pour estre cappitaine d'Orlians, par le conseil de Monseigneur le Regent, lequel nous avoit faict proposition qu'il nous falloit ung cappitaine pour la seureté de la dite ville et du pays d'autour Orlians afin que ung chascun peust labourer ». 
de Cheveaulx répondent à une demande des procureurs, les assurent de leur dévouement ${ }^{80}$ et disparaissent des comptes en mai 1422.

Les trois derniers registres accusent un accroissement certain du passage des familiers et des capitaines du roi Charles ${ }^{81}$. Conseillers, officiers, archevêques, chefs de troupes figurent au titre de dons de vin, de poissons, de chapons et de perdreaux. Un cadeau plus substantiel est offert au Bâtard d'Orléans : 960 1.p. en juin $1421^{82}$; c'est la seule occasion où les comptes marquent pour lui une quelconque distinction, il n'est pas, semble-t-il, encore perçu comme une autorité à consulter ${ }^{83}$.

Les nombreux passages et séjours des gens du roi, loin d'être une protection, sont un souci pour la ville qui doit fournir, outre gite, boissons et nourriture, des hommes d'armes en 1421 et 1425 , et auparavant des canons (en 1419) s4 $^{84}$ Et la ville se sent bien mal récompensée quand elle voit que les faveurs du roi vont à Bourges ${ }^{85}$.

${ }^{80} \mathrm{CC} 548, \mathrm{XXI}$, don à Pierre de Mornay dit Gaulnet : « pour ce qu'il est venu visiter les murs [...] et qu'il a promist aider, conseiller et conforter la ville se les Angloys venoient devant (Orléans)»; ibid., XIX, dîner de Matho de Cheveaulx et de Jean de Beauvillier quand ils vinrent voir ce que l'on « vouloit d'eulx pour la defense de la ville».

${ }^{81}$ C'est dans un mandement de dépenses du 19 novembre 1422 que les comptes de forteresse qualifient pour la première fois Charles de roi : «voyage [...] par devant le Roy nostre sire pour le debat qui estoit au peuple des gros qui ont estes criés a II d.t., pource qu'on ne les voulut prendre, lesquels [envoyés] trouvèrent en chemin qu'on apportoit un mandement de les crier à I d.t $»$.

${ }^{82} \mathrm{CC} 548, \mathrm{IV}$

${ }^{83}$ Le bâtard d'Orléans reçoit des pots de vin, des poissons, du gibier, mais est moins fréquemment cité dans les comptes de forteresse que le maréchal de La Fayette ou le connétable d'Ecosse, peut-être parce qu'il est moins souvent passé par la ville. Les comptes ne mentionnent que deux interventions, la première en 1421 : trois procureurs firent un voyage à Blois «pour voir lettre que Monseigneur le Bastard d'Orlians avoit envoyé pour aler par devant Monseigneur le Regent, pour luy parler de la prison de Monseigneur le duc d'Orlians » (CC 548, I). En 1424, il convoqua les procureurs sur le fait de «l'abstinance de guerre » (CC 549, IX).

${ }^{84} \mathrm{CC} 546, \mathrm{~V}$ : semonce à 58 personnes «pour venir en la chambre où conversent lesdicts procureurs [...] pour oyr lire certaines lettres que Monseigneur le Daulphin envoyoit [...] faisant mention comment on luy envoyast certaine quantité de gens d'armes ». En fait, la ville se fit excuser grâce à l'intervention de Philippe de Vertus (ibid., LIV). Mais quand le Régent réclama des canons, ils lui furent fournis (ibid., LXVI à LXX), tandis que la ville faisait réduire le contingent d'hommes. En effet, au lieu de 20 hommes d'armes et 12 hommes de trait (cf. note 72), « on fist crier par la ville qui qui vouldroit soy mettre aux armes et faire le dict service, qu'il allast par devers les procureurs de la dicte ville d'Orlians et on luy donroit bons gaiges. Et par le dict cry faisant ne fust aucune personne trouvée, ne on n'en peust on finer qui se voulsist armer ou mettre sus aux dit gaiges. Et pour ce fust advisé par les dicts procureurs de composer auxdessus dict chancelier et trésorier de mondict Seigneur à VIII homme, qui valent pour lez dicts VIII hommes à XV francs, VIxx francs ou IIIIxx XVI 1.p. ». CC 548, VI : on fait boire les gens d'armes envoyés au Régent. Ils furent levés nous disent les comptes de ville par Perrin Brullé et partirent le 21 août 1421 (CC 651, XLVI, XVIII).

${ }^{85} \mathrm{CC} 547, \mathrm{XXVII}$ : délégation des Orléanais auprès du comte de Vertus « du fait du mandement comment Monseigneur le Régent avoit mandé à Orlians comment le marchié du bestail feust trois fois la sepmaine à Bourges et non point à Orlians ». 
Alors les procureurs espèrent dans «l'abstinence de guerre », et suivent de très près les tractations qui ont lieu en 1424-1426 entre les Bourguignons, les Bretons et les conseillers de Charles VII. Les Orléanais envoient des messagers, écrivent aux gens de Blois, consultent leurs concitoyens, leur évêque, reçoivent les Blésois, financent Georges de La Trémoille «afin qu'il lui pleust soy en venir pour aler devers Monseigneur de Bourgoigne pour parachever le fait de l'abstinance» en lui allouant 700 écus d'or ${ }^{86}$. Une délégation part à Bourges auprès du Connétable «renouveller certaines lettres par lui données à Chinon, adreçans a Monseigneur de Bourgoigne touchans l'abstinance». Des envoyés descendent la Loire jusqu'à Nantes et y demeurent deux mois "pour sentir se le duc de Bretagne avoit point d'abstinance envers les Angloys, pour lui requerre, de par Monseigneur d'Orlians, que si son pays estoit en abstinance, que il lui pleust d'avoir mémoire des païs de mondict seigneur». Enfin, une petite ambassade part en Angleterre le 26 octobre 1426 auprès du prisonnier «en esperance d'avoir abstinance envers les Angloys et les Bourgoignons $^{87}$ ».

Les trêves sont un des derniers espoirs de la cité, puisque la présence du roi Charles VII en Berry ne permet même pas de vivre en Orléanais sans sauf-conduit. Déjà en 1421, il fallait demander que les «gens d'armes de mondict Seigneur se reculassent du pais d'Orlians affin que l'on peust cueillir l'aoust ${ }^{88} »$. Deux femmes apportèrent en mars 1426 «ung sauf-conduit de Chartres [...] pour semer le mars et pour tous gens de braz estans en Orléanois, Dunois, Vendômois et Beausse. ${ }^{89}$ ». Les pèlerins de Saint-Jacques, pris sans doute pour des espions, ce que certains étaient peut-être, ne pouvait plus sans risque traverser la Loire en 1427 : ils se retrouvaient en geôle ${ }^{90}$.

La forteresse en arrive, ne sachant plus trop sur qui compter, à se retourner vers ses saints. Traditionnellement, la communauté orléanaise faisait annuellement trois processions solennelles, en janvier, mai et août, pour les biens de la terre et, depuis la reprise de la guerre, pour la paix du royaume : on sortait les Corps Saints de l'abbaye de Saint-Aignan, on les promenait par la ville, on faisait des stations aux principales églises, parfois les porteurs passaient la Loire pour aller aux Augustins. Un sermon prononcé par un Mendiant ou un Maître en théologie ajoutait enseignement et réflexion à l'émotion populaire. Les frais étaient en grande partie assumés

${ }^{86} \mathrm{CC} 549$, I et cf. note 61.

${ }^{87} \mathrm{CC} 549, \mathrm{IV}$ : voyage à Bourges ; ibid., XIV : voyage à Nantes ; ibid., XXV : « audit Jaquet le Prestre, le XXVI ${ }^{\mathrm{e}}$ jour d'octobre pour ung present fait à Monseigneur de Mortemar qui alloit devers Monseigneur d'Orlians en Angleterre, et en sa compagnie Hue de Saint-Mars, Maistre Hugues Perrier ». Jaquet Le Prestre était l'infatigable valet de la ville d'Orléans.

${ }^{88} \mathrm{CC} 548$, III ; cf. note 54.

${ }^{89}$ CC 549, XII : elles reçurent XX s.p. Rappelons que le Chartrain était alors aux mains des Anglais. La pénurie en blés est notée dans le même registre (ibid., IV) : voyage du 6 juin 1425 à Bourges, «pour requérir a mondit Seigneur et à la Royne de Cecille que il leur pleust donner lettres adreçans au baillif de Toraine comment il ne fist point destournemens ne empeschemens d'avoir les blés du pays d'embas, pour la grant necessité qui en estoient en ce pays ». Yolande d'Aragon, reine de Sicile, recevait alors les revenus de la Touraine.

${ }^{90} \mathrm{CC} 549, \mathrm{XXXII}$ : «Bonin, lequel fust envoyé à Meung sur Yèvre, porter lettres à Monseigneur le Connestable touchans les pellerins de Saint Jacques qui sont de présent prisonniers ». 
sur les «dépenses communes». En 1421-1423, est-ce un choix du receveur ou décision concertée des procureurs, la «forteresse » participe aux dépenses en payant les six valets qui portent les torches, et en donnant une gratification au clerc chargé du sermon. En 1422, année terrible, il y eut six et non pas trois processions ${ }^{11}$ ! En outre, il y eut au mois d'octobre le passage d'un cordelier qui prêcha au cloître de Sainte Croix (on avait sorti la chaire) et devant la foule assemblée aux halles. Il resta douze jours à l'auberge avec douze compagnons, aux frais des Orléanais, puis se fit raccompagner par deux procureurs jusqu'à Cléry ${ }^{92}$.

Est-ce dire que les élus des Bourgeois de la cité n'espèrent plus qu'en l'aide divine pour obtenir la paix, ou tout au moins l'abstinence de guerre? Je n'irai pas jusque là. En fait, ils n'ont cessé de monter parallèlement leurs deux forteresses, celle de pierre et de bois, de terre et de bronze, et celle des hommes et des alliances. Mais seule la première est tangible. Elle était si puissante et si belle que par deux fois, en 1419 et 1426, les procureurs firent faire une «rouelle» de cire lors des processions ; celle de 1426 était « une présentation de cire ouvrée [...] en une Roelle [...] faicte à Monseigneur Saint-Aignan [... ] où est comprise l'ansainte de la ville d'Orlians ${ }^{93}$ ».

La chute d'une si puissante place n'en serait que plus spectaculaire et ouvrirait à coup sûr la porte du royaume de Bourges. Et bien qu'il n'y eut «pas d'exemple qu'un preux s'attaque aux biens d'un ennemi dont il détient la personne dans ses cachots $^{94}{ }^{2}$, Bedford décida le siège de la ville. Les Orléanais s'enfermèrent dans leur forte muraille et les boulevards creusés avec tant d'opiniâtreté; les hommes d'armes du roi Charles VII ne firent pas mieux que ce que pouvait attendre d'eux la cité bien blasée à leur égard. Mais Orléans tint bon, et le bruit de sa résistance parvint jusqu'à Domrémy alors qu'il était encore temps de la sauver.

\footnotetext{
${ }^{91}$ CC 548, XV : 11 janvier; XX : 27 mai ; XXI : 15 juin ; XXII : 7 juillet ; XXIV : 23 août ; $\mathrm{XXV}: 25$ octobre ; pour la seule année 1422.

${ }^{92} I$ Ibid., XXVI : « hausser la cheze de Sainte Croix [...] porter des selles au cloistre [...] mettre à point les claies que avoient parmi les halles espendues les gens qui oyoient le sermon, esdites halles, du cordellier $»$.

${ }^{93} \mathrm{CC} 547$, VIII : « une roelle de LXXV livres» (mai ou juin 1419). CC 549, XVIII : « une roelle de C livres» (24 juin 1426).

${ }^{94}$ E. Perroy, La guerre de Cent Ans, Paris, 2 éd.,1976, p. 243.
} 